\section{JOURNAL OF PLANT PHYSIOLOGY}

www.elsevier.de/jplph

SHORT COMMUNICATION

\title{
Expansin gene expression and anoxic coleoptile elongation in rice cultivars
}

\author{
Leonardo Magneschi $^{a, *}$, Rasika Lasanthi Kudahettige ${ }^{a}$, Amedeo Alpi $^{\mathrm{b}}$, \\ Pierdomenico Perata ${ }^{a}$
}

\author{
aplant Lab, Scuola Superiore Sant'Anna, Via Mariscoglio 34, 56124 Pisa, Italy \\ ${ }^{b}$ Department of Crop Plant Biology, University of Pisa, Via Mariscoglio 34, 56124 Pisa, Italy
}

Received 15 January 2009; received in revised form 23 March 2009; accepted 23 March 2009

KEYWORDS
Anoxia;
Coleoptile;
Expansin;
Rice;
Oryza sativa

\section{Introduction}

When germinated under anoxia, rice seedlings elongate the coleoptile in order to reach the surface of the water and, as a consequence, transport

\footnotetext{
Abbreviations: EXLA, expansin-like A; EXLB, expansin-like B; EXPA, $\alpha$-expansins; EXPB, $\beta$-expansins; FDR, false discovery rate; qPCR, real-time quantitative PCR.

*Corresponding author at: Plant Lab, Scuola Superiore Sant'Anna, Piazza Martiri della Libertà 33, 56127 Pisa, Italy. Tel.: +390502216553; fax: +390502216532.

E-mail address: magneschi@sssup.it (L. Magneschi).
}

oxygen to the underwater tissues (Alpi and Beevers, 1983). This peculiarity is important for the establishment of rice as a crop (Magneschi et al., 2009).

Coleoptile elongation under anoxia is due to cell expansion. Among the enzymes involved in the process of cell wall loosening, it is likely that expansins play a major role (Cosgrove, 1999; Huang et al., 2000), although other factors regulating cell wall loosening have also been reported (Fry, 1998; Schopfer et al., 2002). Expansins are encoded by a multigene family (Kende et al., 2004) consisting of two sub-families ( $\alpha$ - and $\beta$-expansins, named EXPA 
and $E X P B)$ and by the smaller families of expansinlike $A$ and expansin-like $B$ (EXLA and EXLB), whose biological roles are still unclear (reviewed by Choi et al., 2006). In rice, OsEXPB3, OsEXPB6, OsEXPB11 and OSEXPB12 are induced by treatment with gibberellin, the hormone responsible for rapid internodal elongation in deepwater rice (Lee and Kende, 2001). To date, several positive correlations have been reported among the presence of expansin activity, transcripts, and growth (Vriezen et al., 2000; Lee and Kende, 2001, 2002; Harmer et al., 2002; Lee et al., 2003; Vreeburg et al., 2005; Sasidharan et al., 2008). Under flooding or hypoxic conditions, expansins are often induced by the gaseous hormone ethylene (Jackson, 2008), which is not produced under anoxia (Magneschi et al., 2009). Increases in the mRNA levels of EXPA2 and EXPA4 were observed in submerged-treated internodes of deepwater rice (Cho and Kende, 1997) and in coleoptiles of submerged- or hypoxia-germinated rice seeds (Huang et al., 2000). Interestingly, EXPA4 antisense expression resulted in reduced coleoptile elongation under submergence (Choi et al., 2003). These findings support the hypothesis that EXPA genes may play an important role in mediating elongation in submerged plants. On the other hand, little is known about the role of expansins in the elongation of rice coleoptiles in the complete absence of oxygen (anoxia) (reviewed by Magneschi and Perata, 2009).

Expansin proteins are difficult to purify, and the production of active recombinant expansins and transgenic lines have proved largely unsuccessful (Choi et al., 2003, 2006). A transcriptional approach therefore represents an alternative for the study of this multigene family during anoxic rice germination. Based on microarray data and other gene expression analyses, Lasanthi-Kudahettige et al. (2007) proposed EXPA7, and possibly EXPB12, as candidates for genes involved in the regulation of the anoxic elongation of rice coleoptiles. However, there is no evidence to show a positive correlation between transcript levels of expansin genes and the different abilities of anoxia-induced elongation among rice cultivars.

The aim of this study was to investigate the extent to which expansin genes are induced in anoxic rice coleoptiles and whether different transcript levels of expansin-encoding genes result in the production of longer coleoptiles under anoxia.

\section{Materials and methods}

\section{Plant material}

Rice (Oryza sativa L.) cultivars Arborio Precoce and Lamone (from Ente Nationale Risi, Milan, Italy) caryopses were germinated under aerobic and anoxic conditions as described previously (Lasanthi-Kudahettige et al., 2007). For molecular analyses, coleoptiles were collected every 2 days (from day 4 to day 10) and stored at $-80^{\circ} \mathrm{C}$. Primary leaves were removed from the coleoptiles of air-germinated seedlings (the leaves are absent in anoxia-germinated seedlings). The 2-day-old seedling sample was not used because the coleoptile was too short to be separated from the inner primary leaf.

\section{Microarray data analysis}

Microarray datasets (accession no. GSE6908; http://www.ncbi.nlm.nih.gov/projects/geo) were re-analyzed using the methods described by Lasanthi-Kudahettige et al. (2007). In order to assess differential expression, an empirical Bayesian method (Smyth, 2004) was used to moderate the SE of the estimated log-fold changes. To control $P$-values in a multiple testing context, a Benjamini-Hochberg correction of the false discovery rate (FDR) (Reiner et al., 2003) was applied.

\section{RNA isolation and real-time reverse transcription PCR}

Total RNA was extracted, subjected to DNase treatment, reverse transcribed and used for realtime PCR amplification as previously described (Lasanthi-Kudahettige et al., 2007). The list of primer sequences used in real-time PCR is reported in supplementary Table S1. Glc-3-P dehydrogenase (Os08g03290) was used as a reference. Relative quantification of each individual gene's expression was performed as previously described (LasanthiKudahettige et al., 2007).

\section{Results}

\section{Comparison of anoxic coleoptile elongation in rice cultivars "Arborio Precoce" and "Lamone" and microarray data analysis}

To examine whether the transcript levels of expansin genes correlated with the ability to elongate the coleoptile under anoxia, we selected two Italian rice cultivars with a contrasting anoxic phenotype (Magneschi et al., 2009; Figure 1A). Arborio Precoce was characterized by a long coleoptile under anoxia, whereas the Lamone coleoptile was much shorter ( 72 versus $38 \mathrm{~mm}$ after 10 days of anoxic germination) (Figure 1B). 
In order to obtain a list of expansin-encoding genes to be analyzed in the two rice varieties used in this study, we re-analyzed the microarray datasets (Lasanthi-Kudahettige et al., 2007) to highlight all those expansin genes with a differential expression (Figure 1C).
A

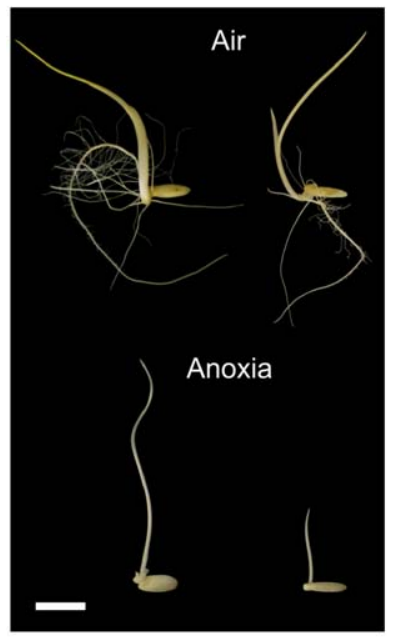

Arborio Precoce Lamone
C

\begin{tabular}{|c|c|}
\hline EXPA7 & $10 X^{* * *}$ \\
\hline EXPA6 & $3 X^{*}$ \\
\hline EXPA5 & $2 X^{*}$ \\
\hline EXPA10 & $-1 X^{*}$ \\
\hline EXPA4 & $-2 X$ ** \\
\hline EXPA1 & $-3 X *$ \\
\hline EXPA2 & $-9 X^{* * *}$ \\
\hline$E X P B 12$ & $9 X^{\star \star \star}$ \\
\hline EXPB4 & $-2 X * *$ \\
\hline EXPB7 & $-2 X^{*}$ \\
\hline EXPB8 & $-2 X^{*}$ \\
\hline EXPB11 & $-3 X^{* * *}$ \\
\hline EXPB3 & $-6 X^{* *}$ \\
\hline EXPB6 & $-10 X * * *$ \\
\hline EXPB2 & $4 X^{* * *}$ \\
\hline
\end{tabular}

0 $169033815071 \quad 8453$
B



Figure 1. Expansin gene expression and anoxic coleoptile elongation of two rice cultivars: (A) seed germination phenotype of 4-day-old aerobic versus anoxic seedlings of Arborio Precoce and Lamone. White bar is equal to $1 \mathrm{~cm}$. (B) Coleoptile length of Arborio Precoce and Lamone seedlings germinated in air $(O)$ and anoxia $(\bullet)$ at $28^{\circ} \mathrm{C}$ in the dark. Data are the means of 30 measurements \pm SD. (C) Expansin-encoding genes reported to be differentially expressed in rice coleoptiles (cv. Nipponbare) by Lasanthi-Kudahettige et al. (2007). Data are expressed as a heatmap showing the signal intensity from the microarray experiment (data are means of two GeneChip experiments) performed on coleoptiles from rice seeds germinated in air and anoxia for 4 days. Fold change is shown on the right-hand side of the heat map. FDR $P$ values: ${ }^{*}>0.05,{ }^{* *} \leqslant 0.05$ and ${ }^{* * *} \leqslant 0.01$.

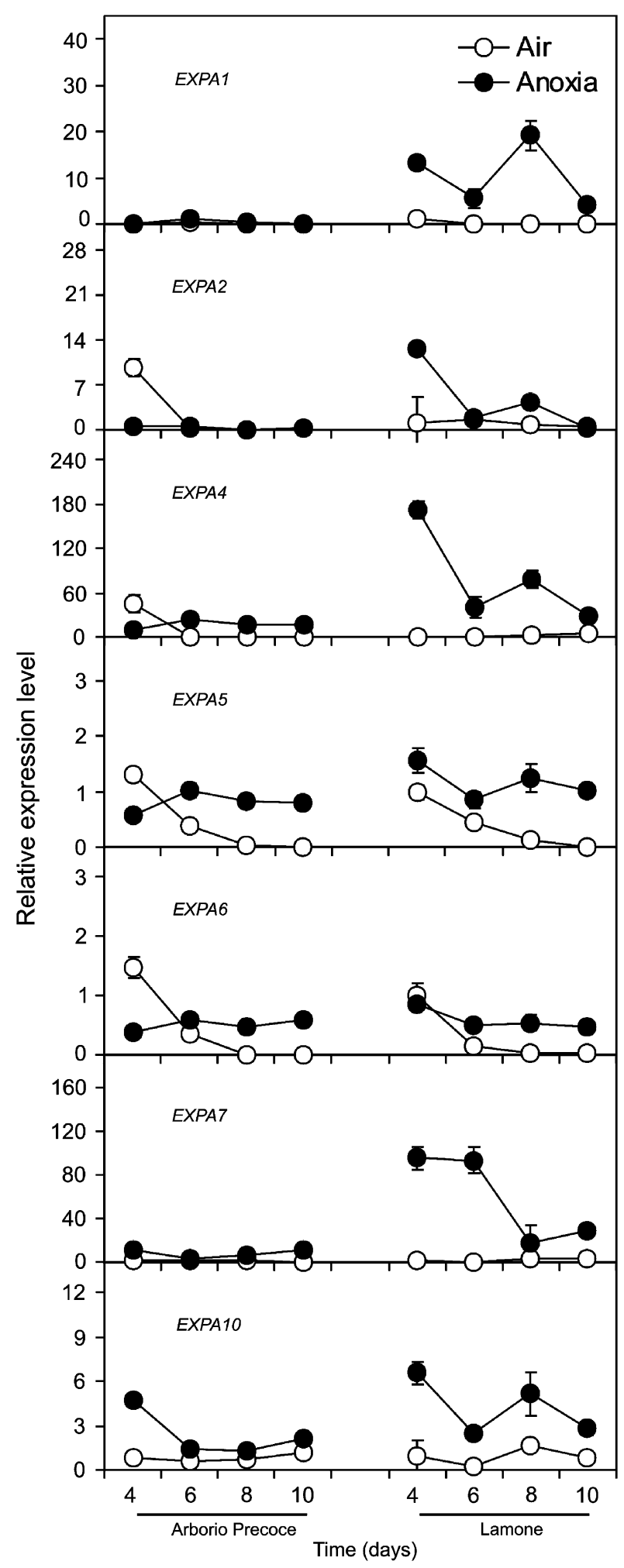

Figure 2. Expression pattern of selected $\alpha$-expansin genes in aerobic $(O)$ and anoxic $(\bullet)$ coleoptiles of rice $\mathrm{cv}$. Arborio Precoce and cv. Lamone grown at $28^{\circ} \mathrm{C}$ in the dark. The expression levels measured by real-time RTPCR are shown (relative expression level: $1=$ expression data from the aerobic coleoptile at day 4 of Lamone). Data are the means of three replicates \pm SD. 


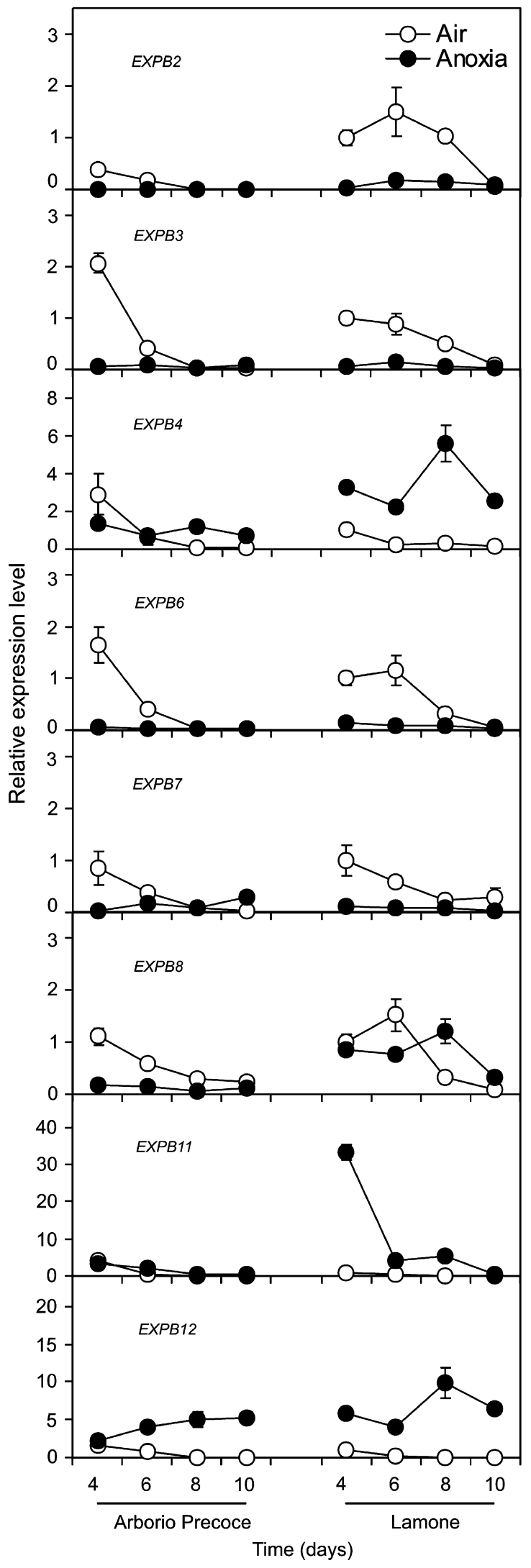

\section{Expression pattern of $\alpha$-expansin genes}

EXPA2, EXPA4 and EXPA7 were found to be the only EXPA showing a FDR $P$-value lower than 0.05 in the cultivar Nipponbare (Figure 1C; LasanthiKudahettige et al., 2007). Real-time reverse transcription PCR analysis showed that EXPA2 and EXPA4 were expressed more under anoxia in Lamone coleoptiles, while Arborio Precoce coleoptiles showed a lower transcript level of EXPA4 in 4-dayold anoxic coleoptiles and a slight up-regulation of the transcripts from the 6th day after imbibition (Figure 2).

EXPA7 has been proposed as a good candidate gene underlying coleoptile elongation under anoxia (Lasanthi-Kudahettige et al., 2007). This gene was also up-regulated under anoxia in the two rice varieties used in this study, but the short-coleoptile cultivar Lamone showed higher induction of EXPA7 transcripts compared to the long anoxic-coleoptile cultivar Arborio Precoce (Figure 2).

EXPA1 was up-regulated over 10 -fold only in 4day-old Lamone coleoptiles. EXPA5 was up-regulated in Nipponbare (Figure 1C), Lamone and Arborio Precoce anoxic coleoptiles (Figure 2). The EXPA6 expression pattern was very similar to EXPA5 in the two cultivars used in this study (Figure 2). EXPA10 expression was unaffected by anoxia in Nipponbare (Lasanthi-Kudahettige et al., 2007), but was up-regulated under anoxia in both Arborio Precoce and Lamone (Figure 2).

\section{Expression pattern of $\beta$-expansin genes}

Six out of eight EXPB showed an FDR lower than 0.05 and only EXPB12 was up-regulated in 4-day-old anoxic Nipponbare coleoptiles (Figure 1C; LasanthiKudahettige et al., 2007).

EXPB2, EXPB3, and EXPB6 were found to be down-regulated in 4-day-old Nipponbare coleoptiles, and this was confirmed in the two cultivars used in this study (Figure 3). EXPB4 and EXPB11 were found to be anoxia up-regulated during anoxic germination in cv. Lamone only (Figure 3). The EXPB12 mRNA level was high under anoxia not only in Nipponbare (Lasanthi-Kudahettige et al., 2007) but also in both Italian cultivars, with comparable

Figure 3. Expression pattern of selected $\beta$-expansin genes in aerobic $(\bigcirc)$ and anoxic $(\odot)$ coleoptiles of rice cv. Arborio Precoce and cv. Lamone grown at $28{ }^{\circ} \mathrm{C}$ in the dark. The expression levels measured by real-time RTPCR are shown (relative expression level: $1=$ expression data from the aerobic coleoptile at day 4 of Lamone). Data are the means of three replicates \pm SD. 
expression patterns between Arborio Precoce and Lamone (Figure 3).

EXPB7 and EXPB8, both down-regulated in 4-dayold coleoptiles of Nipponbare (Figure 1C), showed a lower transcript level 4 days after imbibition in the anoxic coleoptiles of both Arborio Precoce and Lamone (Figure 3).

\section{Discussion}

The mechanisms underlying rapid coleoptile elongation under anoxia are still largely unknown. The anoxic growth rate of the coleoptile, which often exceeds the aerobic growth rate, suggests that proteins such as expansins might be involved (Choi et al., 2006). When analyzing the expression patterns of 15 expansin genes whose expression is affected by anoxia (Figure 1C), we found no relationship between the transcript levels of expansin genes known to be induced by anoxia (Lasanthi-Kudahettige et al., 2007) and anoxic coleoptile elongation. The short-anoxic coleoptile cultivar (Lamone) almost always showed higher levels of transcripts encoding for EXPA (Figure 2) and EXPB (Figure 3) than Arborio Precoce. These data clearly indicate that the transcript levels of expansin genes are not related to the final length of the anoxic coleoptile.

Our results suggest that (i) expansins other than those analyzed are responsible for coleoptile elongation under anoxia, (ii) the mRNA levels measured do not reflect the actual amount of expansin proteins in the coleoptiles, or (iii) expansin gene polymorphisms could explain differences in their ability to elongate the coleoptile. Although the first hypothesis is plausible, it seems unlikely that, in our microarray data analysis, we missed expansin genes that are important for anoxic coleoptile growth 4 days after germination in the cultivar Nipponbare, when the anoxic coleoptile is actively growing (Lasanthi-Kudahettige et al., 2007). However, as reported in this paper, transcript levels of expansin genes vary depending on the rice cultivar (Figures 2 and 3).

Transcript amounts may indeed not reflect the real respective protein levels. Plants respond to low-oxygen stress by regulating gene expression at both the transcriptional and translational levels (Bailey-Serres and Chang, 2005). The higher rate of fermentation observed in Arborio Precoce when compared with Lamone probably indicates that more ATP is available in the long anoxic coleoptile variety (Magneschi et al., 2009), and this may affect the efficiency of cell translational machinery. Finally, single nucleotide polymorphisms in expansin genes among rice cultivars might generate protein variants that differ in terms of efficiency.

In this study, we provided evidence for the enhanced expression of several expansin genes in the anoxic rice coleoptile. The transcript levels of anoxia-enhanced expansins did not, however, correlate with the rate of anoxic coleoptile elongation or with its final length. Further research is clearly needed to shed more light on the role of expansins in coleoptile elongation under anoxia.

\section{Appendix A. Supplementary materials}

Supplementary data associated with this article can be found in the online version at doi:10.1016/ j.jplph.2009.03.008.

\section{References}

Alpi A, Beevers H. Plant Physiol 1983;71:30-4.

Bailey-Serres J, Chang R. Ann Bot 2005;96:507-18.

Cho HT, Kende H. Plant Cell 1997;9:1661-71.

Choi DS, Lee Y, Cho HT, Kende H. Plant Cell 2003;15: 1386-98.

Choi D, Cho HT, Lee Y. Physiol Plant 2006;126:511-8.

Cosgrove DJ. Annu Rev Plant Physiol Plant Mol Biol 1999;50:391-417.

Fry SC. Biochem J 1998;332:507-15.

Harmer S, Orford S, Timmis J. Mol Genet Genom 2002; 268:1-9.

Huang J, Takano T, Akita S. Planta 2000;211:467-73.

Jackson MB. Ann Bot 2008;101:229-48.

Kende $\mathrm{H}$, Bradford KJ, Brummell DA, Cho H-T, Cosgrove DJ, Fleming AJ, et al. Plant Mol Biol 2004;55:311-4.

Lasanthi-Kudahettige R, Magneschi L, Loreti E, Gonzali S, Licausi F, Novi G, et al. Plant Physiol 2007;144:218-31. Lee Y, Kende H. Plant Physiol 2001;127:645-54.

Lee Y, Kende H. Plant Physiol 2002;130:1396-405.

Lee DK, Ahn JH, Song SK, Choi YD, Lee JS. Plant Physiol 2003;131:985-97.

Magneschi L, Perata P. Ann Bot 2009;103:181-96.

Magneschi L, Kudahettige RL, Alpi A, Perata P. Plant Biol 2009.

Reiner A, Yekutieli D, Benjamini Y. Bioinformatics 2003; 19:368-75.

Sasidharan R, Chinnappa CC, Voesenek LACJ, Pierik R. Plant Physiol 2008;148:1557-69.

Schopfer P, Liszkay A, Bechtold M, Frahry G, Wagner A. Planta 2002;214:821-8.

Smyth GK. Stat Appl Genet Mol Biol 2004;3 Article 3.

Vreeburg RAM, Benschop JJ, Peeters AJM, Colmer TD, Ammerlaan AHM, Staal M, et al. Plant J 2005;43: 597-610.

Vriezen WH, De Graaf B, Mariani C, Voesenek L. Planta 2000;210:956-63. 